\title{
QUANTIFYING THE EFFECTS OF EPOXY REPAIR OF REINFORCED CONCRETE PLASTIC HINGES
}

\author{
Kai J. Marder', Kenneth J. Elwood ${ }^{2}$, Christopher J. Motter ${ }^{3}$ \\ and G. Charles Clifton ${ }^{4}$
}

(Submitted May 2019; Reviewed August 2019; Accepted October 2019)

\begin{abstract}
Modern reinforced concrete structures are typically designed to form plastic hinges during strong earthquakes. In post-earthquake situations, repair of moderate plastic hinging damage can be undertaken by filling the crack system with epoxy resin and reconstituting spalled cover concrete. This study uses available experimental test data, including three large-scale ductile beams tested by the authors, to investigate the effects of epoxy repair on the structural behaviour of plastic hinges, with a focus on beam elements. Factors that have been neglected in past studies, including the effects of residual deformations at the time of repair, are given special attention. It is found that epoxy-repaired plastic hinges can exhibit different behaviour from identical undamaged components in terms of stiffness, strength, deformation capacity, and axial elongation. Potential explanations for the observed differences in behaviour are given, and recommendations are made for how these differences can be quantified in order to relate the expected response of an epoxy-repaired plastic hinge to the response that would be calculated for an identical undamaged component.
\end{abstract}

\section{INTRODUCTION}

Early research on the use of epoxies to repair earthquakeinduced damage in reinforced concrete dates back several decades [e.g. 1-3]. Modern performance-based seismic design methodologies, such as FEMA P58 [4], list epoxy injection as a standard repair method for moderate seismic damage, where 'moderate' damage includes cracking, longitudinal reinforcement yielding, and cover concrete spalling. Despite the significant past experimental research and widespread acceptance of the repair method, limited efforts [5] have been made towards quantification of the post-repair behaviour of epoxy-repaired reinforced concrete components. Understanding the effects of epoxy repair of plastic hinging damage is of particular importance, as the formation of plastic hinges during strong earthquakes continues to be the intended behaviour and damage pattern in modern ductile reinforced concrete buildings. Many reinforced concrete moment frames exhibited moderate beam plastic hinging damage following recent earthquakes in New Zealand [6,7], but engineers conducting post-earthquake assessments had little guidance on how to quantify the structural characteristics of buildings repaired by epoxy injection of plastic hinges.

The methodology used in most past experiments on epoxy repair [8-11] was to test an undamaged specimen to an intermediate damage state, repair it, and then retest it using the same loading protocol. This methodology allows for comparison of the stiffness and strength during the repaired and original sections of the test, but since the epoxy repair is conducted prior to failure, the deformation capacity of the undamaged component is unknown and therefore the effects of repair on deformation capacity cannot be assessed. The effects of repair relative to the as-damaged condition (i.e. the performance that would have occurred if the specimen was left unrepaired) also cannot be determined. Furthermore, the residual deformations prior to repair, which may affect the postrepair behaviour, have not been reported in most past test programs.
This study draws on experimental data to make quantifiable recommendations on how to calculate the post-repair response of epoxy-repaired plastic hinges in beam-column elements. Tests conducted by the authors are used to gain insight into the aspects of epoxy repair that have been neglected in past studies, while a dataset that also incorporates previous experiments available in the literature is used to draw further conclusions. The term 'epoxy repair' here refers to epoxy injection of cracks, which may or may not be accompanied by patching of spalled cover concrete using repair mortar. Damage due to degradation of the bond between reinforcement and concrete is implicitly accounted for in this study. However, the experimental dataset generally only covers well-anchored longitudinal reinforcement, such that the findings are not applicable to beam-column joints with depths not meeting modern ductile design criteria. The findings of this study may also be applicable to repairs that use vacuum impregnation to fill cracks with epoxy, although limited data on this method of repair is available [10]. This study is focused on repair of moderate flexural damage, as previously defined. The results are not applicable to repair of damage not consistent with flexural plastic hinging, or repair of severe flexural damage involving crushing of core concrete and buckling or fracture of longitudinal reinforcement.

\section{SUMMARY OF RELEVANT EXPERIMENTAL TESTS}

\section{Beam Tests by Marder et al. [12]}

The test program conducted by the authors involved testing of three epoxy-repaired beams as well as numerous non-repaired beam specimens. A test matrix of all specimens considered in this paper is given in Table 1, and the loading protocols applied to the various specimens are shown in Fig. 1. All beams in the test program were nominally identical and designed to meet the provisions for ductile plastic regions in NZS 3101:2006 [13]. All specimens also meet the criteria for special moment frame beams in ACI 318-19 [14]. The specimens were tested as

\footnotetext{
1 Former Ph.D. Student, Dept. of Civil and Environmental Engineering, University of Auckland, Auckland, New Zealand (corresponding author). Email:kmar702@aucklanduni.ac.nz.

2 Professor, Dept. of Civil and Environmental Engineering, University of Auckland, Auckland, New Zealand.

3 Assistant Professor, Dept. of Civil and Environmental Engineering, Washington State University, Pullman, WA, USA.

4 Associate Professor, Dept. of Civil and Environmental Engineering, University of Auckland, Auckland, New Zealand.
} 
cantilevers. The complete test program is described, and the data are publicly available in the paper by Marder et al. [12] Analysis using the test results was not included in the data paper [12]. Information on the influence of loading protocol on beam response is included in Marder et al. [15], while this paper is focused on epoxy repair of damaged plastic hinges.

Table 1: Test matrix of Marder et al. [12] specimens discussed in this study.

\begin{tabular}{|c|c|c|c|c|}
\hline Specimen & Loading Protocol & $\begin{array}{c}\text { EQ } \\
\text { Peak } \\
\text { Drift }\end{array}$ & Repair & $\begin{array}{c}\text { Axial } \\
\text { Restraint }\end{array}$ \\
\hline MONO & Monotonic & $\mathrm{N} / \mathrm{A}$ & None & None \\
\hline CYC & Cyclic [Fig. 1(a)] & $\mathrm{N} / \mathrm{A}$ & None & None \\
\hline CYC-LER & Cyclic [Fig. 1(a)] & $\mathrm{N} / \mathrm{A}$ & None & $15 \mathrm{kN} / \mathrm{mm}$ \\
\hline CYC-NOEQ & $\begin{array}{l}\text { Modified cyclic } \\
\text { [Fig. 1(d)] }\end{array}$ & $\mathrm{N} / \mathrm{A}$ & None & None \\
\hline LD-1 & $\begin{array}{c}\text { EQ-then-cyclic } \\
\text { [Fig. 1(b)] }\end{array}$ & $1.4 \%$ & None & None \\
\hline LD-1-R & $\begin{array}{c}\text { EQ-then-cyclic } \\
\text { [Fig. 1(b)] }\end{array}$ & $1.4 \%$ & After EQ & None \\
\hline LD-2 & $\begin{array}{l}\text { EQ-then-cyclic } \\
\text { [Fig. 1(c)] }\end{array}$ & $2.2 \%$ & None & None \\
\hline LD-2-R & $\begin{array}{c}\text { EQ-then-cyclic } \\
\text { [Fig. 1(c)] }\end{array}$ & $2.2 \%$ & After EQ & None \\
\hline LD-2-LER & $\begin{array}{c}\text { EQ-then-cyclic } \\
\text { [Fig. 1(c)] }\end{array}$ & $2.2 \%$ & None & $15 \mathrm{kN} / \mathrm{mm}$ \\
\hline LD-2-LER-R & $\begin{array}{c}\text { EQ-then-cyclic } \\
\text { [Fig. 1(c)] }\end{array}$ & $2.2 \%$ & After EQ & $15 \mathrm{kN} / \mathrm{mm}$ \\
\hline
\end{tabular}

Three specimens (named LD-1-R, LD-2-R, and LD-2-LER-R, where "-R" denotes these as repaired) were repaired following initial damaging earthquake-displacement history loadings. The displacement histories were derived from non-linear response history analysis on a ductile moment-frame building subjected to a long duration ground motion (2011 Tohoku earthquake). The peak drift prior to repair was $1.4 \%$ for LD-1-R [Fig. 1(b)] and $2.2 \%$ for LD-2-R and LD-2-LER-R [Fig. 1(c)]. Following repair, the specimens were pushed to failure under a reversedcyclic loading protocol starting with a cycle to a drift slightly larger than the peak drift from the earthquake loading. For each repaired specimen, a nominally identical specimen was subjected to the same initial earthquake loading, left unrepaired, and cycled to failure (specimens LD-1, LD-2, and LD-2-LER). This methodology was intended to allow the effects of repair to be evaluated relative to the same beam left in its damaged state, which reflects a decision faced by building owners and engineers following earthquakes.

For the specimens subjected to earthquake loading, the initial damaging loadings were applied dynamically (loading rates equivalent to that anticipated during earthquake loading) but the subsequent cyclic loading was applied quasi-statically. Additional non-repaired specimens (MONO, CYC, CYC-LER, and CYC-NOEQ) were tested entirely quasi-statically. Various loading protocols, both cyclic (see Fig. 1) and monotonic, were applied to these additional specimens. Specimen CYC-NOEQ is particularly noteworthy as it was subjected to only the cyclic loading portion of the LD-2 loading protocol, as shown in Fig. 1(d). As the specimen boundary conditions and loading are the same as was applied post-repair to specimen LD-2-R, direct comparison can be made between the repaired (LD-2-R) and undamaged (CYC-NOEQ) beams.

The specimens with the suffix -LER were subjected to axial compression in proportion to the development of axial elongation (i.e. a constant stiffness axial spring was utilized), in order to simulate the resistance to beam elongation that can occur in moment frames [16]. The stiffness of the axial restraint resulted in approximately $15 \mathrm{kN}$ of axial compression being induced per $\mathrm{mm}$ of beam elongation. For more information see [12].
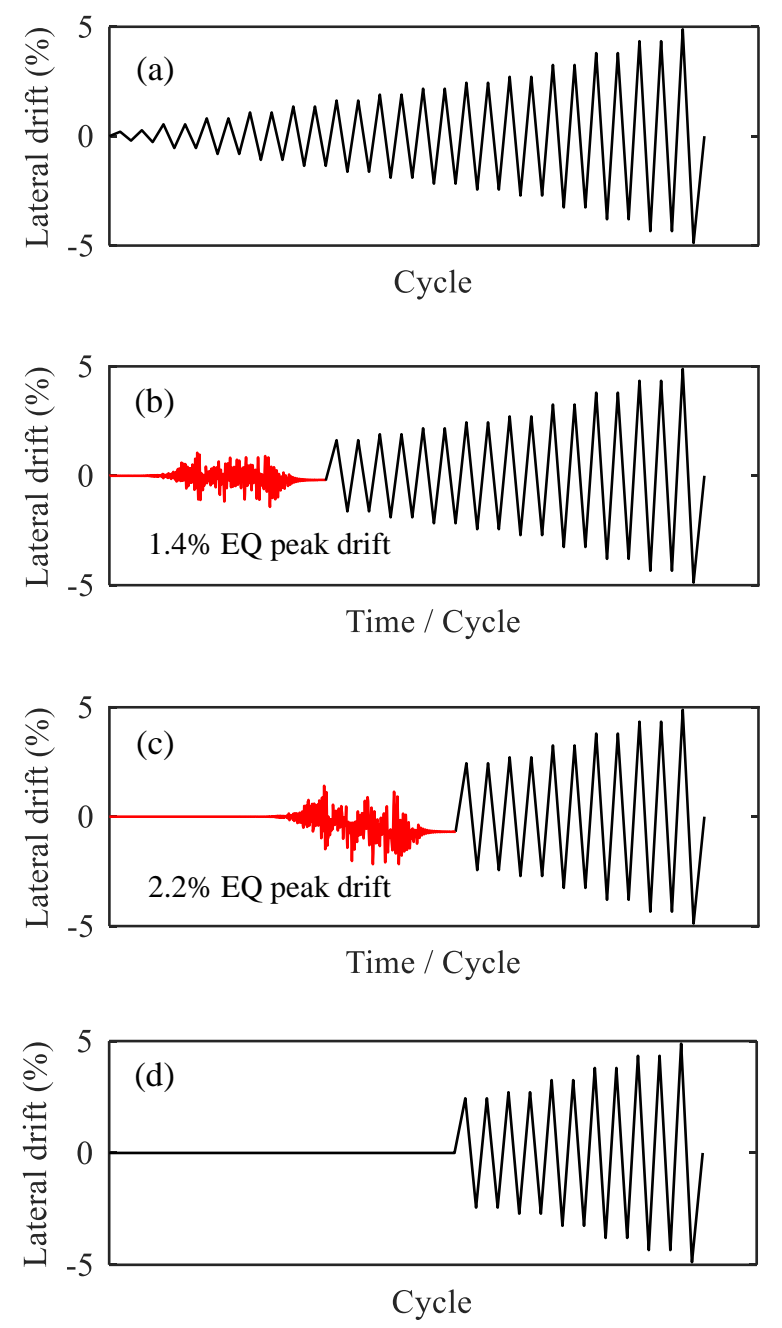

Figure 1: Loading protocols applied to specimens (a) CYC and CYC-LER, (b) LD-1 \& LD-1-R, (c) LD-2 \& LD-2-R and LD-2-LER \& LD-2-LER-R, and (d) CYC-NOEQ. Initial earthquake (EQ) loading shown in red; cyclic loading shown in black.

For the three repaired specimens, photographs of the damage immediately prior to repair are shown in Fig. 2. Photographs of the equivalent specimen pairs at the same point in the test (i.e. immediately following the initial earthquake loading) are also shown. In all specimens, the damage consisted of flexural cracking, longitudinal cracking along the longitudinal reinforcement, and minor delamination of cover concrete. The maximum residual crack widths ranged from $2.5-3.5 \mathrm{~mm}$. The repair consisted of epoxy injection of cracks and replacement of delaminated cover concrete with a high strength repair mortar. The repair was conducted under the supervision of a contractor experienced in epoxy repair of earthquake damage. Despite application of identical loading protocols, the equivalent specimen pairs (e.g. LD-1 and LD-1-R) exhibited different damage patterns following the initial earthquake loadings. The authors attribute this to inherent uncertainty in the development of damage in reinforced concrete plastic hinges, and more information is provided in [15]. 


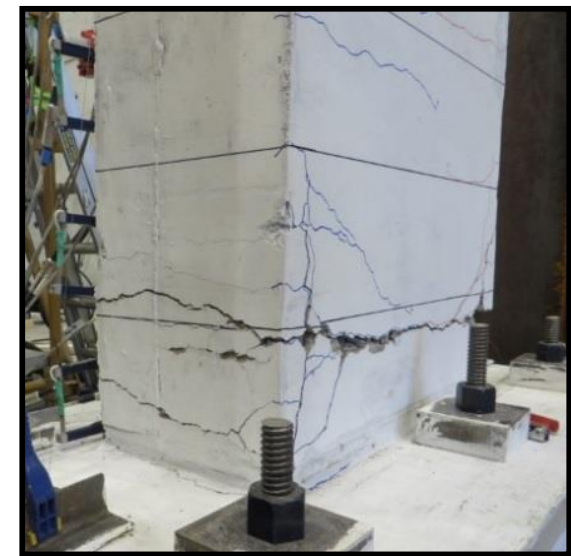

LD-1-R

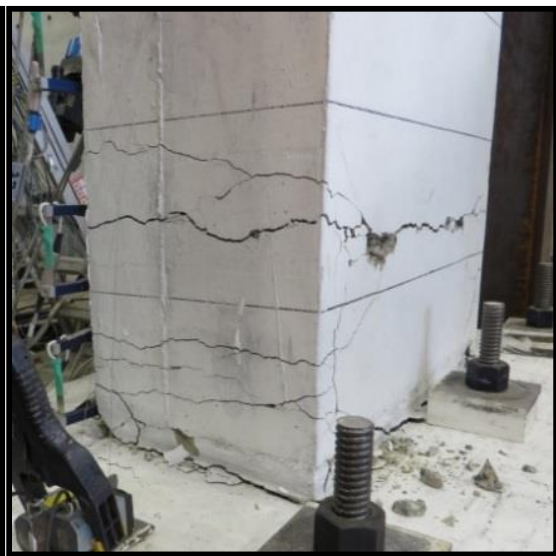

LD-2-R

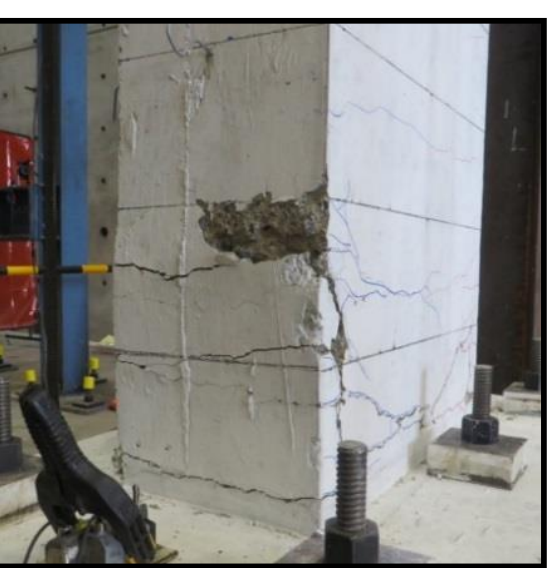

LD-2-LER-R

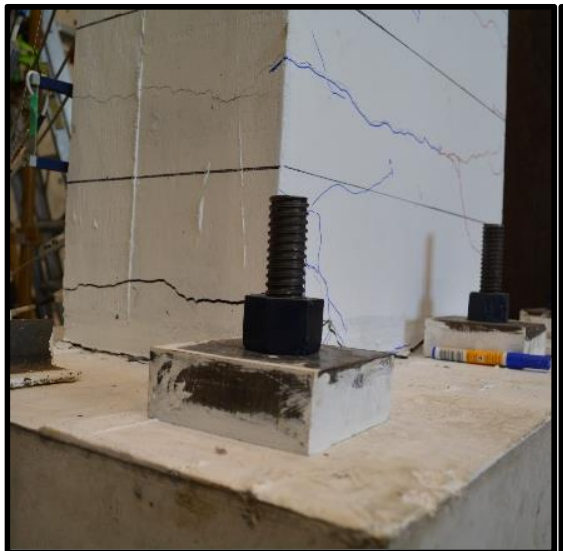

LD-1

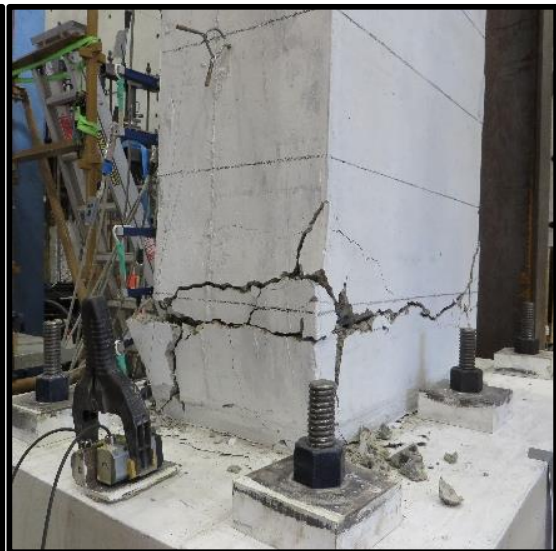

LD-2

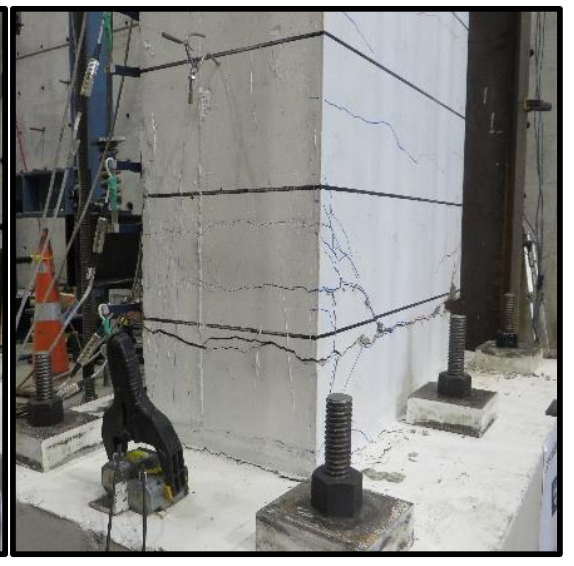

LD-2-LER

Figure 2: Damage states immediately prior to repair for the three repaired beam specimens (top row), and comparison with damage states at the same point in the test for the equivalent unrepaired specimens (bottom row).

\section{Dataset of All Relevant Experiments}

Table 2 lists specimen information for a dataset of fourteen tests on epoxy-repaired reinforced concrete plastic hinges, consisting of eleven tests identified in the literature and the three tests conducted by the authors. Only tests involving epoxy repair of moderately-damaged plastic hinges in beam-column elements subjected to simulated seismic loadings were included in the dataset. Experimental programs involving epoxy repair of specimens that exhibited damage patterns inconsistent with ductile plastic hinging [e.g. 17-19] were omitted. Also not considered are test specimens that were pushed to heavy damage states prior to repair, and required complete reconstitution of the core concrete or replacement of the reinforcement in addition to epoxy repair [e.g. 17,20]. The relevant test specimens identified in the literature include beams $[8,11]$, exterior and interior beam-column subassemblies $[9,10]$, and bridge columns [21]. The damage states of the specimens prior to repair varied, but in all cases yielding of the longitudinal reinforcement had occurred. The Lehman et al. test
[21] is unique among the available test data in that axial load $\left(0.07 A_{g} f_{c}{ }_{c}\right)$ was applied to the specimen. Lehman et al. [21] also tested an additional nominally identical column to failure, without repair, which will be used below for further assessment of the effectiveness of epoxy-injection repair.

Table 3 provides a summary of the effects of epoxy repair on the secant stiffness to yield, the ultimate strength, the cycle-tocycle energy dissipation, and the deformation capacity. In some cases, numerical data were not available and these parameters had to be estimated from load-deformation plots. Visually estimated values of the displacement ductility prior to repair were used as a consistent deformation metric due to the difficulty of extracting accurate beam drifts from the Lee et al. [9] and French et al. [10] results. Where quantitative data could not be obtained with confidence across all specimens, qualitative measures are used (e.g. for the energy dissipation and deformation capacity data). The comparison in cycle-tocycle energy dissipation was only considered up to the onset of strength degradation. 
Table 2: Experimental dataset of epoxy-repaired plastic hinges: specimen information.

\begin{tabular}{|c|c|c|c|c|c|c|}
\hline Study & Component type & $\begin{array}{l}\text { Specimen } \\
\text { name }\end{array}$ & $\begin{array}{l}\text { Cross-section } \\
(\mathrm{mm})^{1}\end{array}$ & $\begin{array}{l}\text { Long. reo. } \\
\text { ratio }(\%)^{1}\end{array}$ & $\begin{array}{c}\text { Transv. reo. } \\
\text { ratio }(\%)^{1}\end{array}$ & $\begin{array}{l}\text { Shear span to } \\
\text { depth ratio }{ }^{1}\end{array}$ \\
\hline $\begin{array}{l}\text { Celebi and } \\
\text { Penzien [8] }\end{array}$ & Beam & $\begin{array}{c}\text { Celebi-5 } \\
\text { Celebi- } 7^{2} \\
\text { Celebi- }^{2} \\
\text { Celebi- } 10^{2}\end{array}$ & $380 \times 180$ & 1.3 & $\begin{array}{l}0.9 \\
0.5 \\
0.9 \\
0.9\end{array}$ & $\begin{array}{l}5.1 \\
3.7 \\
3.7 \\
3.7\end{array}$ \\
\hline Lee et al. [9] & $\begin{array}{l}\text { Exterior beam- } \\
\text { column subassembly }\end{array}$ & $\begin{array}{l}\text { Lee-1 } \\
\text { Lee-3 }\end{array}$ & $250 \times 210$ & Asymmetric & $\begin{array}{l}0.6 \\
0.3\end{array}$ & 4.7 \\
\hline French et al. [10] & $\begin{array}{l}\text { Interior beam-column } \\
\text { subassembly }\end{array}$ & $\begin{array}{l}\text { French-RVI } \\
\text { French-RPI }\end{array}$ & $510 \times 310$ & 0.7 & 0.5 & 4.3 \\
\hline $\begin{array}{l}\text { Lehman et al. } \\
\text { [21] }\end{array}$ & Bridge column & $\begin{array}{l}\text { Lehman- } \\
415 \mathrm{MR}^{2}\end{array}$ & 610 (circular) & 1.5 & 0.7 & 4.0 \\
\hline $\begin{array}{l}\text { Cuevas and } \\
\text { Pampanin [11] }\end{array}$ & Beam & $\begin{array}{l}\text { Cuevas-2 } \\
\text { Cuevas-3 }\end{array}$ & $1100 \times 575$ & 0.6 & 0.5 & 2.3 \\
\hline Marder et al. 12] & Beam & $\begin{array}{c}\text { LD-1-R }{ }^{2} \\
\text { LD-2-R } \\
\text { LD-2-LER- }{ }^{2}\end{array}$ & $720 \times 320$ & 0.6 & 0.4 & 3.6 \\
\hline
\end{tabular}

${ }^{1}$ For tests on beam-column subassemblies, the listed data corresponds to the beams, which were the primary location of plastic hinging in all cases. ${ }^{2}$ Specimen used in deformation capacity analysis.

Table 3: Experimental dataset of epoxy-repaired plastic hinges: key results.

\begin{tabular}{|c|c|c|c|c|c|c|}
\hline $\begin{array}{l}\text { Specimen } \\
\text { name }\end{array}$ & $\begin{array}{l}\text { Approx. ductility } \\
\text { demand before } \\
\text { repair }\end{array}$ & $\begin{array}{l}\text { Approx. drift } \\
\text { demand before } \\
\text { repair (\%) }\end{array}$ & $\begin{array}{c}\text { Secant } \\
\text { stiffness to } \\
\text { yield ratio }{ }^{1}\end{array}$ & $\begin{array}{c}\text { Ultimate } \\
\text { strength ratio }{ }^{1}\end{array}$ & $\begin{array}{c}\text { Energy } \\
\text { dissipation ratio }{ }^{1}\end{array}$ & $\begin{array}{l}\text { Deformation } \\
\text { capacity ratio }\end{array}$ \\
\hline Celebi-5 & 3.5 & 3.0 & $N / A^{2}$ & $1.11^{3}$ & Similar ( 0.9-1.1) & $\mathrm{N} / \mathrm{A}^{4}$ \\
\hline Celebi-7 & 4 & 3.0 & $N / A^{2}$ & $1.21^{3}$ & Similar ( 0.9-1.1) & Reduced \\
\hline Celebi-9 & 4 & 3.0 & $N / A^{2}$ & $1.19^{3}$ & Similar ( 0.9-1.1) & Reduced \\
\hline Celebi-10 & 5 & 3.0 & 1.1 & 1.17 & Similar ( 0.9-1.1) & Reduced \\
\hline Lee-1 & 4 & $\begin{array}{l}\text { Beam drift not } \\
\text { isolated }\end{array}$ & 0.9 & 1.28 & Similar ( 0.9-1.1) & $\mathrm{N} / \mathrm{A}^{4}$ \\
\hline Lee-3 & 4 & $\begin{array}{l}\text { Beam drift not } \\
\text { isolated }\end{array}$ & 1.0 & 1.24 & Similar ( 0.9-1.1) & $N / A^{4}$ \\
\hline French-RVI & 4 & $\begin{array}{l}\text { Beam drift not } \\
\text { isolated }\end{array}$ & 0.85 & 1.01 & Reduced $(<0.9)$ & $\mathrm{N} / \mathrm{A}^{4}$ \\
\hline French-RPI & 4 & $\begin{array}{l}\text { Beam drift not } \\
\text { isolated }\end{array}$ & 0.9 & 1.05 & Reduced $(<0.9)$ & $N / A^{4}$ \\
\hline Lehman-415MR & 3.5 & 3.1 & 0.5 & 1.0 & Similar ( 0.9-1.1) & Similar $(\sim 1.0)$ \\
\hline Cuevas-2 & 1.2 & 0.6 & 1.0 & 1.05 & Similar ( 0.9-1.1) & $\mathrm{N} / \mathrm{A}^{4}$ \\
\hline Cuevas-3 & 2.2 & 1.1 & 0.9 & 1.05 & Similar $(\sim 0.9-1.1)$ & $N / A^{4}$ \\
\hline LD-1-R & 3.4 & 1.4 & 0.85 & 1.04 & Similar ( 0.9-1.1) & Increased $(>1.1)$ \\
\hline LD-2-R & 5.4 & 2.2 & 0.76 & 1.07 & Increased $(>1.1)$ & Similar ( 1.0) \\
\hline LD-2-LER-R & 5.4 & 2.2 & 0.85 & 1.07 & Similar $(\sim 0.9-1.1)$ & Similar ( 1.0) \\
\hline
\end{tabular}

${ }^{1}$ The ratio refers to the value of the parameter for the repaired response, divided by the value for the original response prior to repair (except in the cases of Lehman-415MR, LD-1-R, LD-2-R, and LD-2-LER-R, where the comparisons are against nominally identical undamaged specimens).

${ }^{2}$ The stiffness values before and after repair are not comparable due to different loading rates.

${ }^{3}$ The strength ratio neglects the spike at yield that occurred in the original unrepaired specimens due to dynamic loading rates.

${ }^{4}$ The test procedure did not allow for comparison between the deformation capacity of repaired and undamaged specimens. 
(a)

LD-2

(unrepaired after EQ)

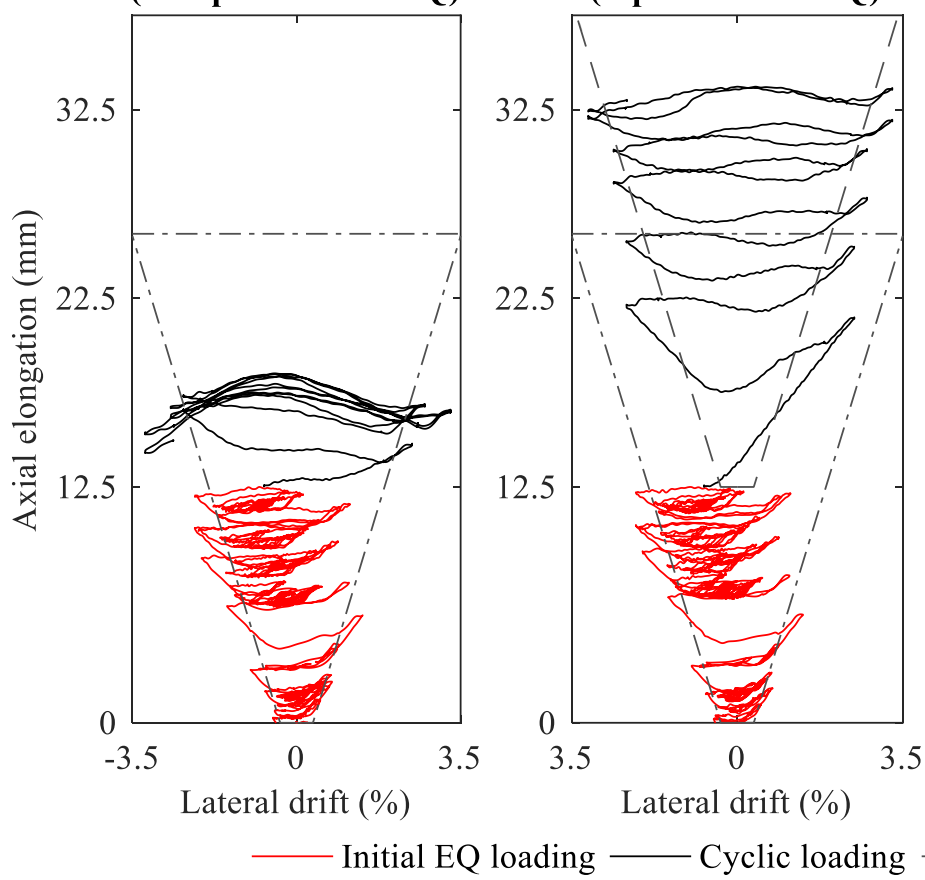

(b)

LD-2-R (repaired after EQ)
IOR TO

The presence of residual axial elongation at the time of repair can cause an increase in the maximum cumulative elongation (i.e. the sum of the pre-repair residual elongation and the postrepair elongation) of a repaired plastic hinge, relative to the maximum elongation that would be likely to occur if no repair was conducted. The experiments identified in the literature did not typically report the residual deformations prior to repair, but this increase in cumulative elongation was evident in the repaired beam specimens tested by the authors.

Fig. 3 shows how the post-repair elongation versus drift response of specimen LD-2-R [Fig. 3(b)] was similar to that of an undamaged specimen subjected to an identical cyclic loading protocol (CYC-NOEQ, shown in [Fig. 3(c)]). The post-repair elongation of specimen LD-2-R was cumulative to the residual elongation of approximately $12.5 \mathrm{~mm}$ that was measured following the initial earthquake loading. The equivalent unrepaired specimen LD-2 [Fig. 3(a)] exhibited the same residual elongation of $12.5 \mathrm{~mm}$ following the initial earthquake loading, but considerably less elongation during cyclic loading ( $5 \mathrm{~mm}$ versus $20 \mathrm{~mm})$, as shear sliding deformations became dominant and prevented further elongation from developing.

In order to quantify the cumulative elongation in a repaired plastic hinge, it is recommended that the post-repair elongation be considered as equivalent to what would develop in an identical undamaged component. Eq. (1) was introduced in NZS 3101:2006 Amendment 3 [13] as a conservative method of calculating the maximum expected elongation for a given rotation demand in a reversing plastic hinge not subjected to axial load. Fig. 3 shows that this equation conservatively overpredicts the elongation for the unrepaired specimens, but underpredicts the post-repair elongation of specimen LD-2-R. The maximum expected cumulative elongation in a repaired plastic hinge for a given (post-repair) rotation demand can be conservatively estimated by simply adding the measured (c)

CYC-NOEQ

(undamaged prior to cycling)

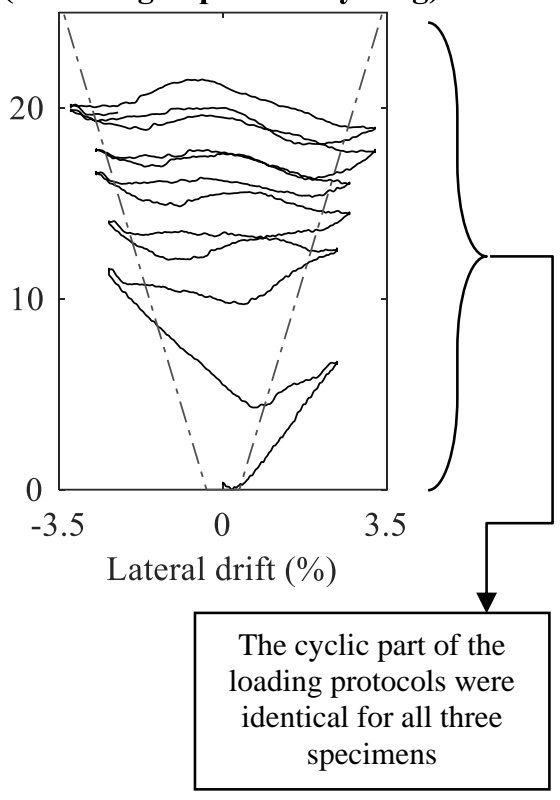


subjected to the same initial earthquake loading. As a result of the reduced residual elongation, the impact of repair on the maximum cumulative elongation that developed in specimen LD-2-LER-R was relatively minor $(4 \mathrm{~mm}$ more than in specimen LD-2-LER). For practical purposes, this implies that it may be possible to neglect any increase in cumulative elongation due to repair in members with enough restraint to axial elongation (e.g. columns supporting gravity loads). In beams, the restraint to axial elongation provided by the surrounding structure is not readily quantified, and it is therefore recommended that beams be considered free to elongate for the purpose of estimating an upper bound on cumulative elongation.

If damage in a repaired plastic hinge re-develops within the original plastic hinge zone, any increase in total elongation must be accompanied by an increase in longitudinal reinforcement strain. This could have effects on both the strength (due to strain hardening) and deformation capacity of the repaired plastic hinge. The magnitude of additional strain that has the potential to occur in the longitudinal reinforcement of a repaired plastic hinge can be approximated by assuming the residual elongation at the time of repair is uniformly spread across an estimated plastic hinge length, as shown in Eq. (3). The plastic hinge length in Eq. (3) should be determined using equivalent plastic hinge length models intended for use at or near the ultimate capacity (e.g. $0.5 h_{b}$ ), and not the actual equivalent plastic hinge length at the time of repair $\left(l_{p}=\right.$ $\left.\theta_{p} / \varphi_{p, \max }\right)$.

$$
\varepsilon_{\text {avg }}=e_{\text {resid }} / l_{p}
$$

where $\varepsilon_{\text {avg }}$ is the average residual strain across $l_{p}$ at the time of repair, $e_{\text {resid }}$ is the residual elongation at the time of repair, and $l_{p}$ is the equivalent plastic hinge length (e.g. $l_{p}=0.5 h_{b}$ ). $\varepsilon_{a v g}$ can then be added to the maximum strain expected to develop during a second earthquake, $\varepsilon_{\max }$, in order to determine the maximum cumulative strain that has the potential to occur in the repaired plastic hinge. The assumptions inherent in this calculation are illustrated in Fig. 4.

Equivalent plastic hinge length models (e.g. $l_{p}=0.5 h_{b}$ ) are not applicable prior to the development of strain hardening in the longitudinal reinforcement, which allows the strain to redistribute along the plastic hinge. However, in ductile members, the ultimate capacity occurs after a well-distributed plastic hinge has developed, and it is therefore here assumed that localized fluctuations in the strain profile along the length of the longitudinal reinforcement at the time of repair are not of significance, as a more distributed strain profile would be expected to develop upon further loading. Eq. (3) is applicable in any situation where ductile detailing will ensure a welldistributed plastic hinge will form, even if only a single crack is present at the time of repair. However, it must be emphasised that Eavg is not a measure of the actual peak strain in the reinforcement at the time of repair, which will typically be higher.
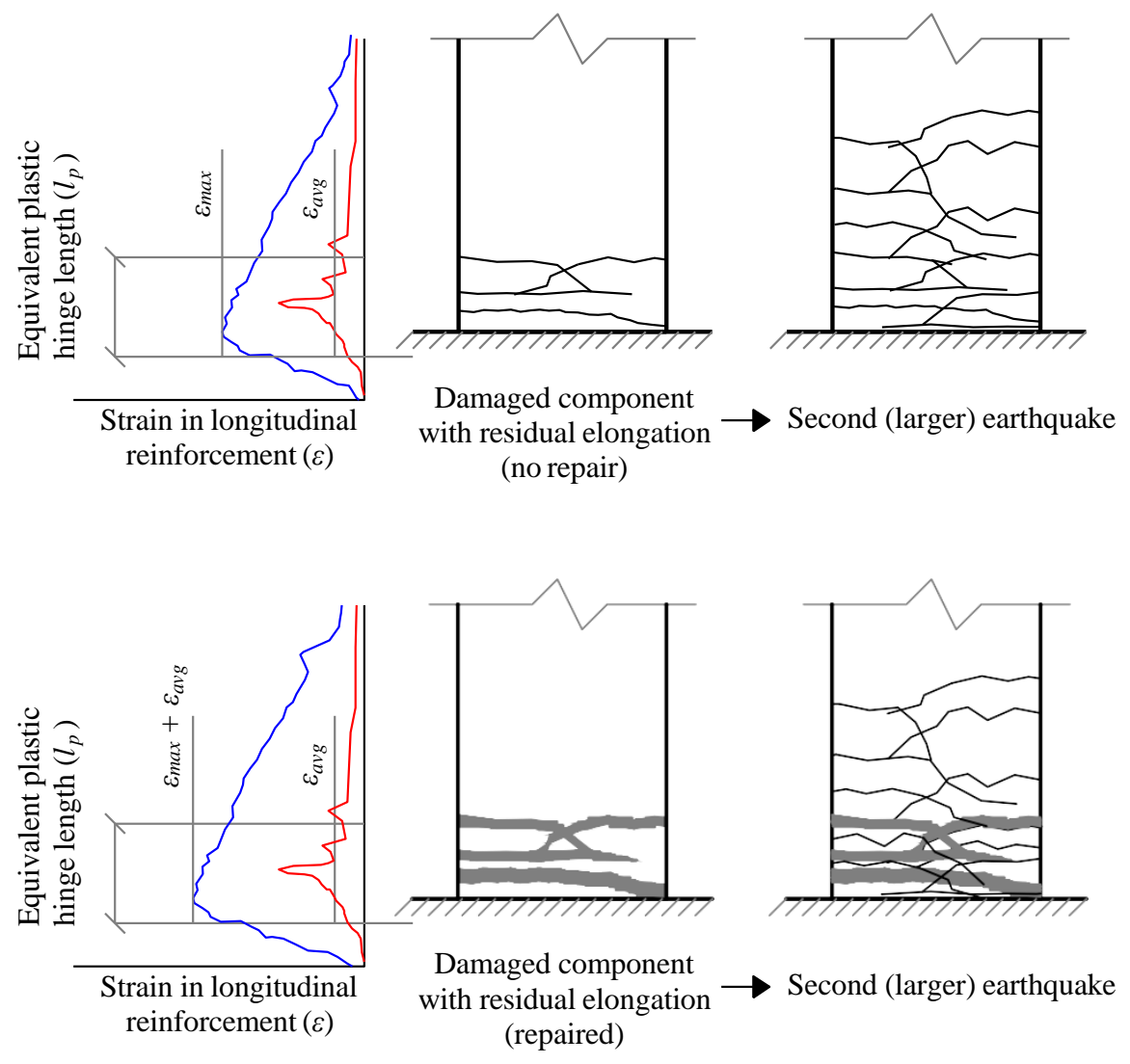

Strain at time of repair

Strain after second (larger) earthquake

Figure 4: Theoretical effect of residual elongation at the time of repair on the maximum longitudinal reinforcement strain in a second earthquake. 
The presence of residual rotation at the time of repair (and the associated residual strain gradient) can similarly alter the strain demands in a repaired plastic hinge, relative to what would occur in an unrepaired equivalent. In the repaired beam specimens tested by the authors, however, residual rotations between $0.26-0.75 \%$ were found to have no impact on the postrepair deformation capacity. It is here recommended that residual rotations (i.e. member drift or chord rotation) of $0.75 \%$ or below are ignored when assessing post-repair residual capacity of plastic hinges. Further experiments are required to determine the efficacy of epoxy injection repair on plastic hinges with larger residual rotations. Nonetheless, this effect is expected to be modest in practical situations, as plastic hinges with significant residual rotation are only likely to occur in buildings with residual drifts of $1 \%$ or above, and such buildings are unlikely to be economical to repair [4].

\section{POST-REPAIR DAMAGE DEVELOPMENT}

Fig. 5 illustrates three possible scenarios with regards to the reoccurrence of plastic hinging damage in an area that has previously been repaired. Both plastic hinge relocation [Fig. $5(\mathrm{a})]$ and re-formation of damage within the repaired plastic hinge [Fig. 5(c)] can have consequences with regards to the post-repair residual capacity, with the underlying reasons being largely mutually exclusive. An intermediate case, where damage partially occurs within the repaired area and partially relocates, is also possible [Fig. 5(b)]. Based on descriptions of damage in all test programs from the dataset described previously, where damage re-forms in the same region, epoxyfilled cracks typically remain closed, and new cracks open in the adjacent concrete.

The test specimens of the dataset (Table 2) exhibited a variety of post-repair damage progressions. Celebi and Penzien [8], Lee et al. [9], and French et al. [10] all reported some degree of plastic hinge relocation or lengthening following repair in one or more specimens. Lehman et al. [21] did not report any lengthening of the plastic hinge after repair. Cuevas and Pampanin [11] reported some relocation of the plastic hinge; however, these results were more challenging to interpret as the specimens had additional reinforcement close to the column face which resulted in shifting of the plastic hinge regardless. In the specimens tested by the authors, more distributed damage, i.e. the case illustrated by Fig. 5(b), occurred following repair. In the three repaired specimens, measurable cracks (cracks with widths greater than $0.2 \mathrm{~mm}$ ) developed up to an average distance of $540 \mathrm{~mm}$ from the beam end, compared to an average of $430 \mathrm{~mm}$ in non-repaired specimens. However, this more distributed damage in the repaired specimens did not result in the formation of different ultimate failure mechanisms compared to that observed in non-repaired specimens.

Plastic hinge relocation [Fig. 5(a)] occurs if the strength of the repaired area increases by an amount sufficient to prevent further damage from developing in that region. A potential complication of plastic hinge relocation is increased curvature demands for a given drift demand, due to a reduced shear span length. Re-formation of plastic hinge damage within the repaired area [Fig. 5(c)] occurs if the strength of the repaired area does not increase enough to force hinge relocation. This can result in higher longitudinal reinforcement strains (due to additional cumulative elongation within the hinge, as previously discussed) and the re-loading of reinforcement that was previously subjected to inelastic demands. Previously yielded reinforcement can have altered properties as a result of strain ageing, a time-dependent phenomenon that can cause an increase in the strength and reduction in strain capacity of certain steels that have previously strain hardened [23]. Furthermore, low-cycle fatigue can cause reinforcing steel to fracture at a lower strain than it would under monotonic loading [24]. In either case (relocated or non-relocated), an increase in shear force and other associated actions (e.g. joint shear and column actions for a beam plastic hinge in a moment frame) occurs as a result of any increased moment capacity in the repaired region.

In some cases, it may be desirable to force relocation of the plastic hinge to occur, in order to protect the previously yielded longitudinal reinforcement. This may be achievable through retrofit with fibre-reinforced polymers or jacketing techniques [e.g. 18], but such retrofit is outside the scope of this paper and would still increase shear force and other associated actions. (a) Plastic hinge relocates away from repaired area

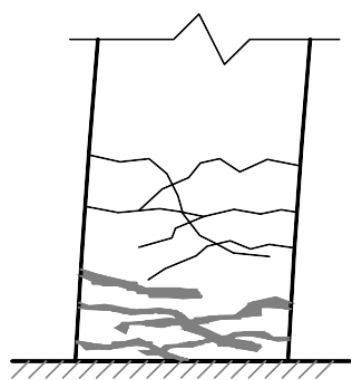

- Increased rotation demands for a given drift demand

- Inelastic demand occurs on reinforcement that has not previously been subjected to large strains (b) Plastic hinge lengthens, with damage both within and outside repaired area

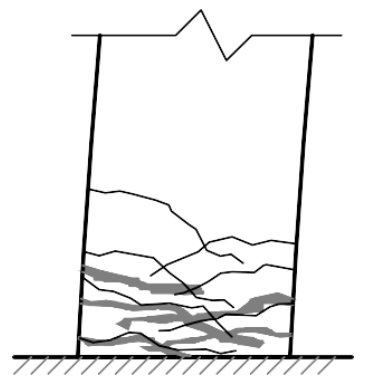

- Intermediate between cases (a) and (c) (c) Plastic hinge re-forms in repaired area

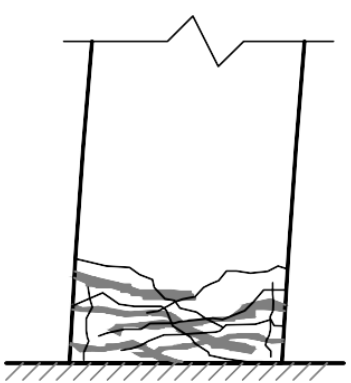

- Increased strains in reinforcement if residual elongation present at time of repair

- Reinforcement may have reduced strain capacities due to low-cycle fatigue and strain ageing 


\section{STIFFNESS}

Eleven of the tests in the dataset (Table 2) had data conducive to assessing the ratio of secant stiffness to yield for post-repair and equivalent undamaged cases. As shown in Table 3, ten of the specimens had stiffness ratios ranging from approximately $0.8-1.1$, with the Lehman-415MR specimen being an outlier with a stiffness ratio of approximately 0.5 . The Lehman415MR specimen was a circular bridge column with axial compression while all other specimens involved plastic hinging in a rectangular beam without axial load (except the cyclic compression induced in the one axially-restrained specimen LD-2-LER-R). Lehman et al. [21] attributed the reduced stiffness to degradation of the concrete stiffness and strength, and degradation of the bond capacity between the concrete and reinforcing steel. Another possible hypothesis for the lower stiffness is that the axial load may have caused closure of cracks at which the longitudinal reinforcement had previously yielded, limiting the ability of the epoxy resin to penetrate these cracks. It is recommended that additional experimental work be conducted on epoxy repair of ductile reinforced concrete columns subjected to axial compression to further investigate these hypotheses.

In Fig. 6, the ratios of repaired to undamaged secant stiffness to yield are compared against the approximate maximum applied displacement ductility prior to repair. It can be seen that the level of displacement ductility that the specimens were subjected to prior to repair is not correlated with the ratio of repaired to undamaged stiffness. This lack of correlation indicates that the demands incurred prior to repair do not have to be considered when estimating the post-repair stiffness, a convenient outcome given the uncertainty in estimating the prerepair demands. It is here recommended that epoxy repair of plastic hinges in beams can be conservatively assumed to restore a secant stiffness to yield that is $80 \%$ of what would be calculated for an equivalent undamaged beam. This is consistent with the recommendations made by FEMA 306 for epoxy injection of moderately damaged reinforced concrete walls or coupling beams [5]. A bounding analysis, with an upper bound stiffness approximately equal to that of the undamaged beam, may be appropriate in some circumstances.

Previous studies have shown that stiffness degradation in reinforced concrete plastic hinges is partially a function of the ductility demand [25]. Fig. 6 demonstrates that the post-repair stiffness is not correlated with the ductility demand prior to repair. The absolute increase in stiffness due to repair, therefore, increases with the ductility demand prior to repair. Fig. 7 shows the shear force versus lateral drift response during the first $1 / 4$ cycle of cyclic loading following the initial earthquake loading for the repaired and unrepaired equivalent specimen pairs tested by the authors. Table 4 shows the corresponding values for the secant stiffness to $0.8 M_{n}$, where $M_{n}$ is the nominal flexural strength of the beams calculated as per NZS 3101:2006 [13] using nominal material strengths. The percentage increase in stiffness due to repair of specimen LD$1-\mathrm{R}$, relative to LD-1, was $60 \%$, compared to over $140 \%$ increase in the stiffness of the repaired LD-2 and LD-2-LER specimens, which were subjected to higher ductility demands prior to repair (5.4 versus 3.4).

\begin{tabular}{|c|c|c|c|}
\hline $\begin{array}{l}\text { Specimen } \\
\text { pair }\end{array}$ & $\begin{array}{c}\text { Repaired } \\
\text { specimen } \\
\text { stiffness } \\
(\mathrm{kN} / \mathrm{mm} / \\
\left.\text { ratio } E_{c} l_{g}\right)\end{array}$ & $\begin{array}{c}\text { Equivalent } \\
\text { unrepaired } \\
\text { specimen } \\
\text { stiffness } \\
(\mathrm{kN} / \mathrm{mm} / \\
\left.\text { ratio } E_{c} l_{g}\right)\end{array}$ & $\begin{array}{c}\text { Percentage } \\
\text { increase in } \\
\text { stiffness }\end{array}$ \\
\hline LD-1-(R) & $10.3 / 0.23$ & $6.3 / 0.14$ & $60 \%$ \\
\hline LD-2-(R) & $9.2 / 0.20$ & $3.7 / 0.08$ & $150 \%$ \\
\hline LD-2-LER-(R) & $10.3 / 0.23$ & $4.3 / 0.09$ & $140 \%$ \\
\hline
\end{tabular}

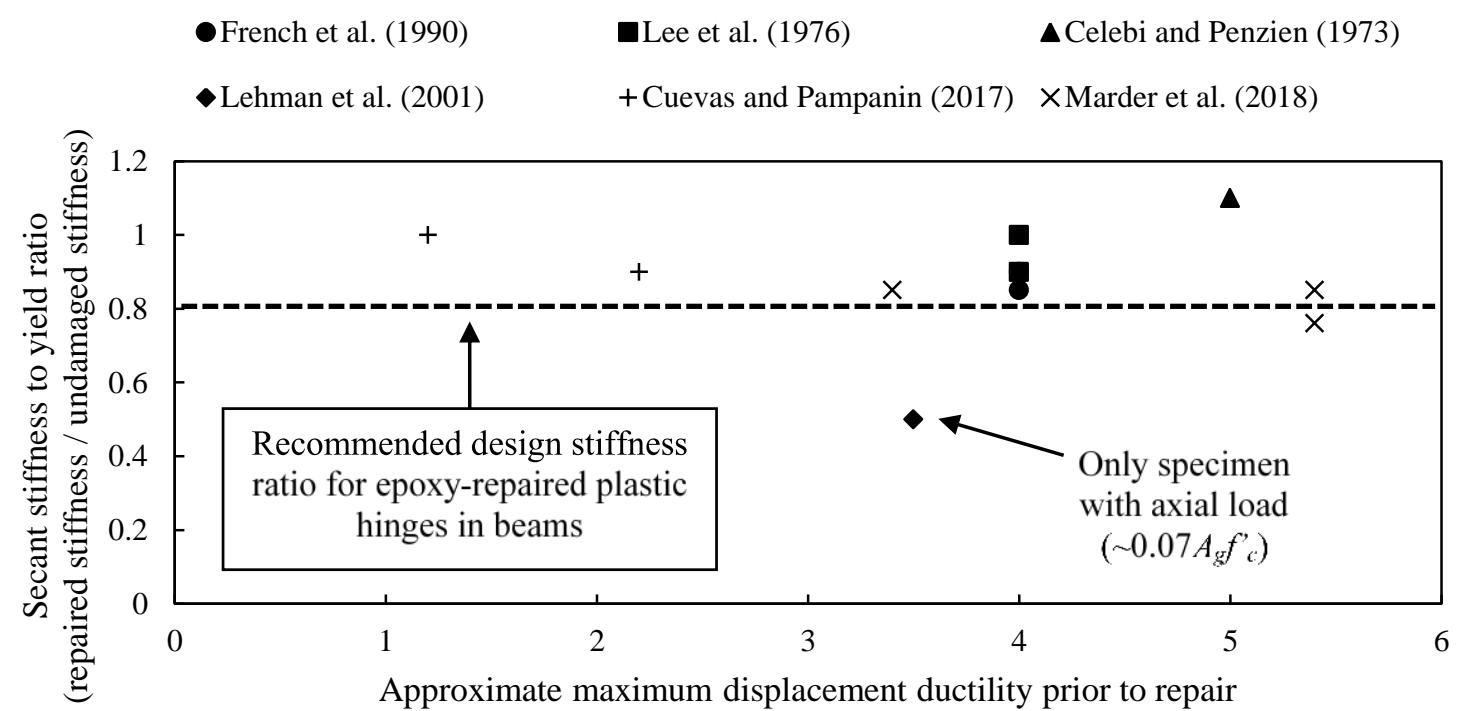

Figure 6: Ratio of repaired to undamaged stiffness versus the displacement ductility prior to repair for eleven test specimens. 

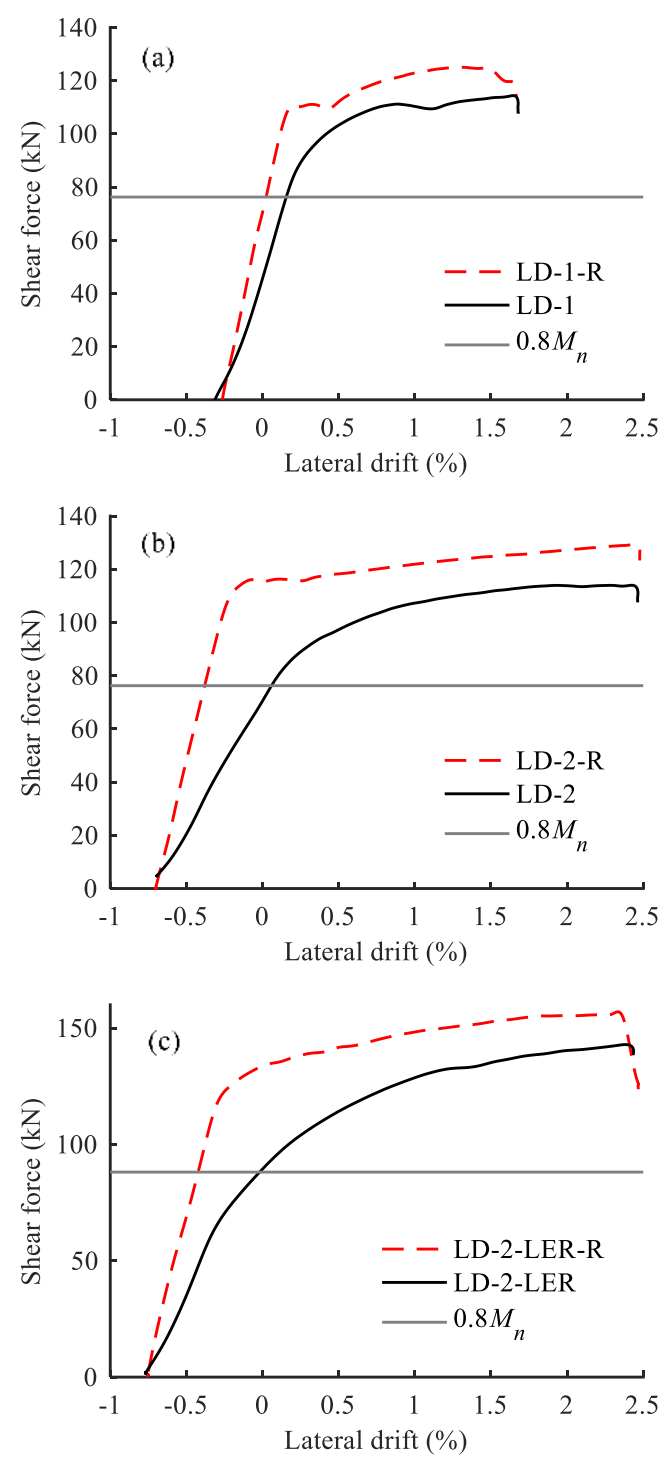

Figure 7: First $1 / 4$ cycle after initial earthquake loadings in repaired and unrepaired equivalent specimen pairs: (a) LD$1-R \& L D-1,(b) L D-2-R \& L D-2$, and (c) LD-2-LER-R \& LD-2-LER.

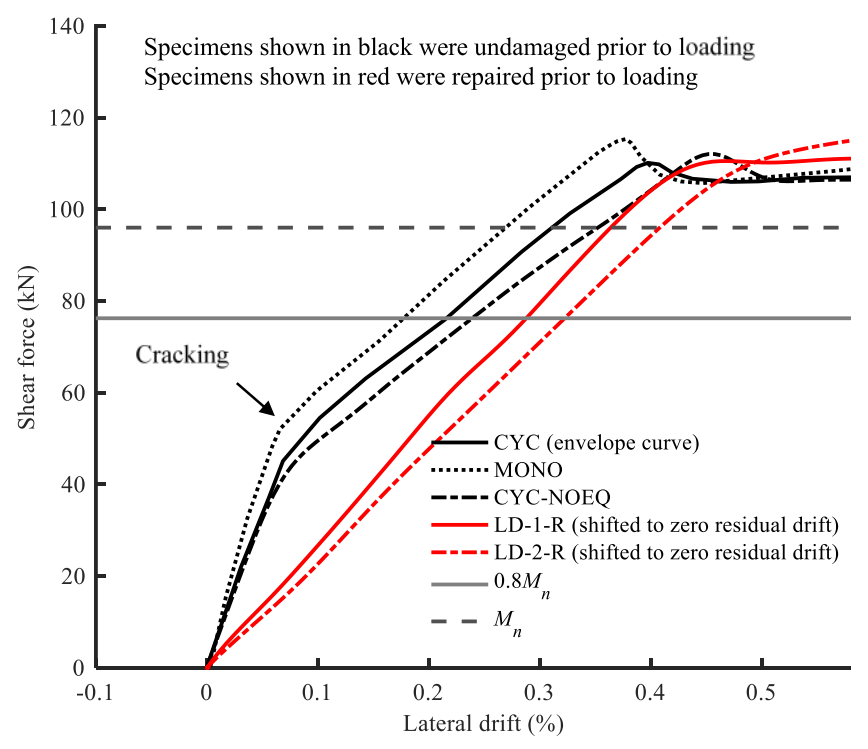

Figure 8: Comparison of initial stiffness between repaired and undamaged nominally identical beam specimens.
Epoxy repair is not able to restore tension stiffening behaviour in reinforced concrete, as epoxy is not able to be injected into small cracks (typically in the range of $0.2 \mathrm{~mm}$ or below). Fig. 8 shows the shear force versus lateral drift response during the first $1 / 4$ cycle of loading for repaired specimens LD-1-R and LD$2-\mathrm{R}$, compared against the equivalent response in three nominally identical undamaged specimens (CYC, MONO, and CYC-NOEQ). Fig. 8 clearly illustrates the lower initial stiffness for the repaired specimens due to the inability to recover the uncracked stiffness. Note that the recommendation to use $80 \%$ of the stiffness of undamaged beams for the post-repair stiffness (Fig. 6) is intended to provide an appropriate effective secant stiffness to yield, such that it is most relevant for use in analysis when the moment demands are greater than or equal to $M_{n}$. Fig. 8 indicates a larger stiffness reduction should be considered when moment demands are less than $M_{n}$.

\section{STRENGTH}

The ratio of ultimate flexural strength between the repaired and undamaged specimens in the dataset (Table 2) ranged from 1.01.3, with an average of 1.1 (Table 3). In Fig. 9, these strength ratios are compared against the approximate maximum applied displacement ductility prior to repair. Similar to the finding for stiffness, the displacement ductility prior to repair was not strongly correlated with the post-repair strength. These data indicate that epoxy-repaired plastic hinges can be expected to have moment capacities at least as high as the original reinforced concrete section, regardless of the demands incurred prior to repair (provided there is only moderate flexural damage, as previously defined).

The large increases in flexural strength that were observed in some specimens are of concern, as a higher than expected beam moment capacity could alter the hierarchy of yielding and consequently the sway mechanism, which could lead to brittle failure. It is here assumed that strength increases in repaired plastic hinges are largely due to increased longitudinal steel strength, which could be a result of strain ageing or higher levels of strain hardening due to increased cumulative elongation. These sources of strength increase are above those accounted for in standard overstrength factors used in capacity design of new buildings [26,27]. Both strain ageing and increased cumulative elongation occur as a result of prior plastic deformation, which results in the conclusion that high deformation demands prior to repair are required in order for increased post-repair strength to occur. This is reflected in Fig. 9 although limited data at low deformation demands are available. The epoxy itself is unlikely to cause a significant increase in member strength because cracks tend to reform in the adjacent concrete.

The degree of strain ageing in any of the repaired specimens in the dataset from Table 2 is unknown, and therefore direct analysis regarding the effects of strain ageing on the post-repair strength is not possible. In the three repaired beam specimens tested by the authors, post-repair testing took place no later than 14 days after the initial damaging loading, and therefore strain ageing is not expected to have had a significant effect [28]. Previous studies have shown that strain ageing can cause strength increases of up to $15 \%$ [28-30]. The injection of epoxy does not have any effect on the degree of strain ageing in reinforcement.

The Celebi and Penzien [8] and Lee et al. [9] test specimens exhibited strength increases of between $10-30 \%$, relative to the original response, while all other repaired specimens exhibited strength increases no greater than $7 \%$. It is suspected that strain ageing of longitudinal reinforcement contributed to the strength increases in the Celebi and Penzien [8] and Lee et al. [9] test programs. Certain alloying metals, notably vanadium, can prevent strain ageing [31], but the Lee et al. [9] test program 
used a longitudinal reinforcement grade of 40ksi (275MPa), likely to have been plain mild steel. Celebi and Penzien [8] did not report the grade of reinforcement used, but the test program was conducted before the first publication (1974) of the ASTM A706 low-alloy reinforcement standard in the United States. In light of the considerable strength increases in these test specimens, and in lieu of better data, use of a $1.15 f_{y}$ factor is recommended to account for the effects of strain ageing for the purposes of assessing overstrength capacities of repaired plastic hinges with susceptible steel. This recommended factor is in addition to standard reinforcing steel overstrength factors intended to convert between nominal and expected strengths and the degree of strain hardening expected in the design earthquake (e.g. 1.35fy in [13]). Further research is required to validate the proposed $1.15 f_{y}$ strain ageing factor.

Despite a lack of strain ageing, the repaired beam specimens tested by Marder et al. [12] exhibited higher strengths than comparable non-repaired specimens in all cases. The strength increases due to the repair were modest, ranging from 4 to $7 \%$ relative to the average non-repaired specimen strength. This increase can be attributed to the increase in strain hardening due to residual elongation at the time of repair (i.e. $\varepsilon_{a v g}$, as calculated by Eq. (3)). Assuming an idealized bi-linear strain hardening response of the reinforcement, the strain hardening ratio $b$ can be calculated by Eq. (4).

$b=\frac{\left(f_{u}-f_{y}\right)}{\left(\varepsilon_{u}-\varepsilon_{y}\right) E_{s}}=\frac{\left(f_{u}-f_{y}\right)}{\left(\varepsilon_{u} E_{s}-f_{y}\right)}$

where $f_{u}$ is the ultimate stress, $f_{y}$ is the yield stress, $\varepsilon_{u}$ is the strain at ultimate stress (i.e. the uniform strain), $\varepsilon_{y}$ is the yield strain, and $E_{s}$ is the elastic modulus.
In the strain hardening branch of the bi-linear curve, the theoretical increase in strength $(\Delta f)$ for a given additional strain $(\Delta \varepsilon)$ can be calculated by Eq. (5).

$\Delta f=b E_{s} \Delta \varepsilon$

The longitudinal reinforcing steel used in the authors' test program had mean measured properties of $f_{y}=300 \mathrm{MPa}, f_{u}=$ $440 \mathrm{MPa}$, and $\varepsilon_{u}=0.22$. Taking $E_{s}=200 \mathrm{GPa}$ gives a corresponding $b$ of 0.0032 . Table 5 shows the calculated increases in longitudinal reinforcement strength due to residual elongation at the time of repair (i.e. $\Delta \varepsilon=\varepsilon_{\text {avg }}$ ) for the three repaired beam specimens. The percentage increases in longitudinal reinforcement strength correlate well with the increases in strength of the repaired beam specimens. The increase in beam strength is under-predicted in specimen LD2-LER-R, but the post-repair strength increase in that specimen was partially due to increased cumulative elongation resulting in higher axial compression forces induced by the restraint system.

This detailed method of calculating the potential increase in post-repair flexural strength as a result of increased strain hardening requires knowledge of the in-situ material properties to determine a suitable value of the strain hardening ratio $b$; however, this may not be feasible in practice. An alternative approach is to obtain an upper-bound estimate of the potential strength increase by making a conservative assumption for the value $b$. Using the upper characteristic $f_{u} / f_{y}$ ratio and the lower characteristic uniform strain from AS/NZS 4671 [32] in Eq. (4), $b$ can be conservatively estimated as $b=0.005$ for $300 \mathrm{E}$ and $b$ $=0.01$ for $500 \mathrm{E}$.

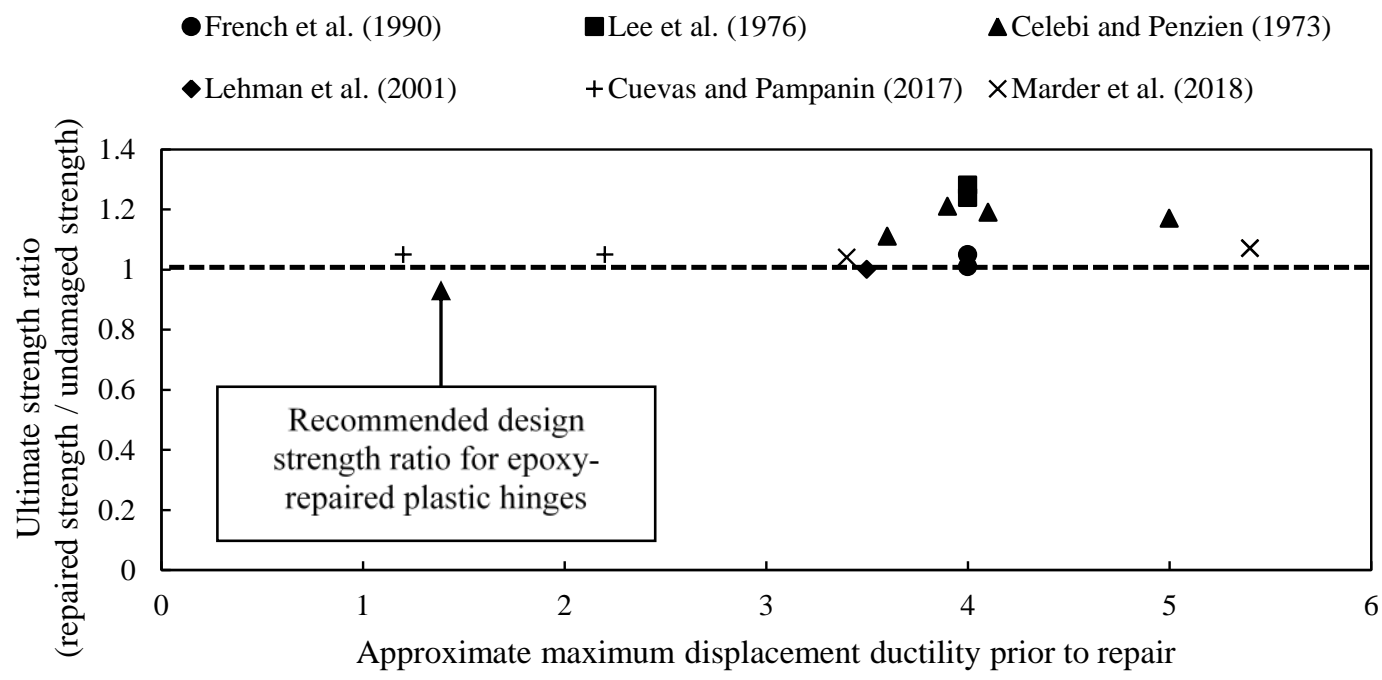

Figure 9: Ratio of repaired to undamaged flexural strength versus the displacement ductility prior to repair for fourteen test specimens.

Table 5: Calculated increase in longitudinal steel strength due to increased strain hardening in repaired beam specimens.

\begin{tabular}{cccccc} 
Specimen & $\boldsymbol{\varepsilon}_{\text {avg }}{ }^{1}$ & $\boldsymbol{b}$ & $\begin{array}{c}\boldsymbol{\Delta} \boldsymbol{f}=\boldsymbol{b} E_{s} \boldsymbol{\varepsilon}_{\text {avg }} \\
(\mathrm{MPa})\end{array}$ & $\begin{array}{c}\text { Percentage } \\
\text { increase } \\
\boldsymbol{\Delta} \boldsymbol{f} / \boldsymbol{f}_{\boldsymbol{y}}\end{array}$ & $\begin{array}{c}\text { Experimental } \\
\text { beam strength } \\
\text { increase (\%) }\end{array}$ \\
\hline LD-1-R & 0.017 & 0.0032 & 11 & 3.7 & 4 \\
LD-2-R & 0.034 & 0.0032 & 22 & 7.3 & 7 \\
$\begin{array}{c}\text { LD-2-LER- } \\
\text { R }\end{array}$ & 0.014 & 0.0032 & 9 & 3.0 & $7^{2}$ \\
\hline
\end{tabular}

${ }^{1}$ Calculated using Eq. (3) with $l_{p}=0.5 h_{b}=360 \mathrm{~mm}$.

${ }^{2}$ This strength increase was partially a result of increased axial compression. 
In summary, the following factors should be considered when determining the flexural overstrength of a repaired beam:

a) Ratio of expected to nominal steel stress at yield

b) Expected strain hardening in the design earthquake

c) Strain hardening in previous earthquake according to Eq. (5)

d) Strain ageing of grade 300 reinforcement $\left(1.15 f_{y}\right.$ recommended)

Factors (a) and (b) are implicitly included in the recommended overstrength factors specified in NZS 3101 [26]. Additionally, factors (c) and (d) should be included when assessing the overstrength of a repaired beam with moderate damage. Furthermore, movement of the plastic hinge, as described in Figure 5, should be considered a possibility; however, further research is required to identify the conditions which are likely to lead to movement of the plastic hinge.

\section{ENERGY DISSIPATION}

The specimens of the dataset (Table 2) generally exhibited comparable energy dissipation characteristics before and after repair. Fig. 10 shows the energy dissipation in each half-cycle for the equivalent repaired and unrepaired specimen pairs tested by the authors, as well as the average cycle-to-cycle energy dissipation of all non-repaired specimens with the same axial restraint conditions. The repaired specimens all had increased (15 to $25 \%$ higher) energy dissipation in the first cycle immediately following repair. However, the energy dissipation characteristics of specimens LD-1-R and LD-2-LER-R quickly degraded to be similar to that of the non-repaired specimens.
The cycle-to-cycle energy dissipation in specimen LD-2-R remained higher than the average energy dissipation in the nonrepaired specimens throughout the test. This increased energy dissipation in specimen LD-2-R can be attributed to the lower magnitude of shear deformations (and associated reduced pinching of the hysteretic response) that occurred in specimen LD-2-R, as compared to most other specimens. However, this response may have been due to variability in damage progression, rather than the repair, as it did not occur in the other two repaired specimens.

The two interior beam-column subassemblies tested by French et al. [10] both exhibited a post-repair reduction in cycle-tocycle energy dissipation of as much as $20 \%$, relative to the original response. French et al. attributed this reduction in energy dissipation to a degradation of anchorage bond in the joint, and an inability of the epoxy resin to penetrate far enough into the joint to restore the bond strength. The joint width used in the French et al. test program was $380 \mathrm{~mm}$, or 15 times the longitudinal bar diameter $\left(d_{b}\right)$, which is less than the recommended minimum joint width of $20 d_{b}$ in ACI 318-19 [14] and the recommended minimum joint width in NZS 3101 from 1982 [33] onward. The narrow joint width likely resulted in a more severe anchorage degradation than would occur in beamcolumn joints detailed to modern seismic provisions. Considering that all other specimens in the dataset did not exhibit a reduction in energy dissipation after repair, it is assumed that, provided that modern detailing and anchorage requirements are met, epoxy-repaired plastic hinges typically dissipate a similar amount of energy as equivalent undamaged components.

(a)

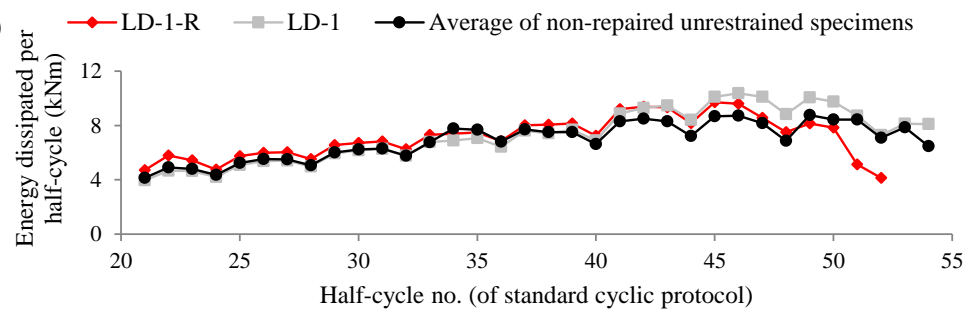

(b)

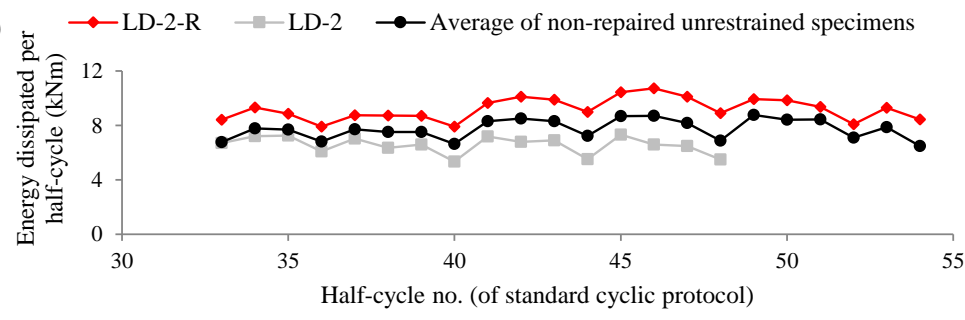

(c)

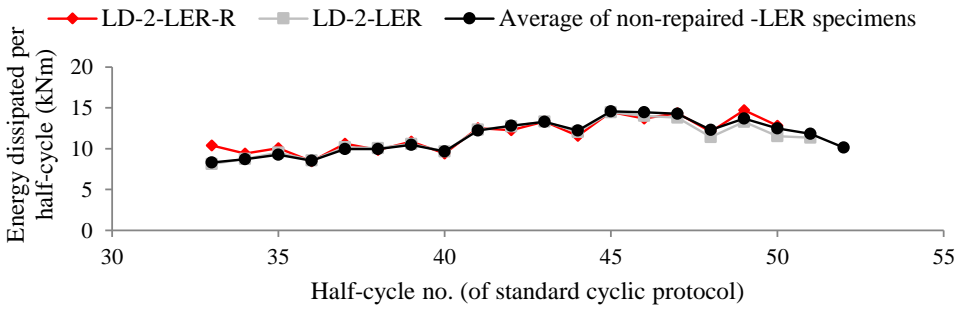

Figure 10: Cycle-to-cycle energy dissipation of the three repaired specimens tested by the authors (a) LD-1-R, (b) LD-2-R, and (c) LD-2-LER-R, and comparison with energy dissipation of equivalent non-repaired specimens. 


\section{DEFORMATION CAPACITY}

Seven of the test specimens in the dataset (identified in Table 2) were tested in a manner that allowed conclusions to be drawn with regards to deformation capacity, either by having equivalent non-repaired specimens for comparison, or due to failure of the repaired specimen before completion of the same loading protocol applied prior to repair. The three beam specimens tested by the authors and the Lehman et al. [21] bridge column had deformation capacities at least as high as those that occurred in equivalent non-repaired specimens. However, three of the four beam specimens tested by Celebi and Penzien [8] failed due to longitudinal reinforcement fracture at a lower deformation demand than was applied prior to repair. Potential causes of reductions in deformation capacity of repaired plastic hinges, relative to undamaged hinges, are discussed below.

If plastic hinge relocation takes place [Fig. 5(a)], the curvature induced in the relocated hinge due to a post-repair drift demand would be higher than that induced in the original hinge if subjected to the same drift demand, assuming the plastic hinge length remains the same. This can be readily accounted for by modifying the geometrical relationships between drift and local curvature or rotation to consider the expected new location of the plastic hinge. It is assumed that standard seismic assessment calculations, unmodified for damage or repair, are appropriate for determining the curvature or rotation capacity of relocated plastic hinges. This is because the majority of the inelastic demand on the longitudinal reinforcement occurs on steel that has not previously been subjected to significant strains. Special attention should be given to the transverse reinforcement detailing of the member, as relocated plastic hinges could cause yielding to spread outside of the region that is specially detailed for ductile behaviour.

If plastic hinge relocation does not take place [Fig. 5(c)], residual elongation at the time of repair can cause an increase in cumulative elongation within the hinge and an associated increase in longitudinal reinforcement strain [as calculated by Eq. (3)]. Reinforcement within the repaired hinge would also be subject to any reduction in strain capacity or fatigue life due to strain ageing and low-cycle fatigue phenomena. Epoxy injection may mitigate these issues to some degree, as epoxy is generally effective at keeping injected cracks closed, thus shifting the location of highest longitudinal reinforcement strains. However, due to varying degrees of strain penetration on either side of a crack and the inability to quantify the distance of a new crack from the original epoxy-injected crack, it is recommended that shifting of localised strains due to epoxy injection be neglected.

Previous research [15] has shown that the number of loading cycles applied at or below $2 \%$ drift does not typically result in a reduced deformation capacity in ductile plastic hinges, based on tests of beams and columns with length (or height) to depth ratios of 2.2 to 5.7 (note that this study did not consider the effects of epoxy repair or strain ageing). This is further evidenced in Fig. 11, where the post-repair hysteretic response of specimen LD-2-R is compared with the response of undamaged specimen CYC-NOEQ. The application of the initial damaging long duration earthquake displacement history to specimen LD-2-R did not result in earlier low-cycle fatigue failure of reinforcement, relative to undamaged specimen CYCNOEQ. Low-cycle fatigue is also indirectly accounted for in modern deformation capacity acceptance criteria (e.g. ASCE 41-17), which are calibrated against test specimens subjected to standard cyclic loading protocols, which typically impose many more cycles than a component experiences in a typical earthquake [34]. Direct consideration of any reduction in longitudinal reinforcement capacity due to low-cycle fatigue effects is therefore considered unnecessary for the purposes of assessing the deformation capacity of epoxy-repaired plastic hinges.

Potential reductions in strain capacity due to strain ageing or residual elongation at the time of repair may need to be considered when assessing deformation capacity. However, a reduction in the strain capacity of longitudinal reinforcement will not necessarily alter the deformation capacity of a plastic hinge. The beams tested by the authors used AS/NZS 4671 [32] grade $300 \mathrm{E}$ longitudinal reinforcement with a measured $\varepsilon_{u}$ of approximately 0.22 . As the ageing period was not sufficient for strain ageing to occur, the only potential source of reduced strain capacity was the residual elongation at the time of repair. The reduction due to residual elongation at the time of repair can be calculated as previously discussed [Eq. (3)]. Using Eq. (3), the repaired beams had $\varepsilon_{\text {avg }}$ values of only 0.014 to 0.034 at the time of repair. It is therefore unsurprising that the deformation capacities of the repaired specimens were unaffected by the residual elongation at the time of repair.

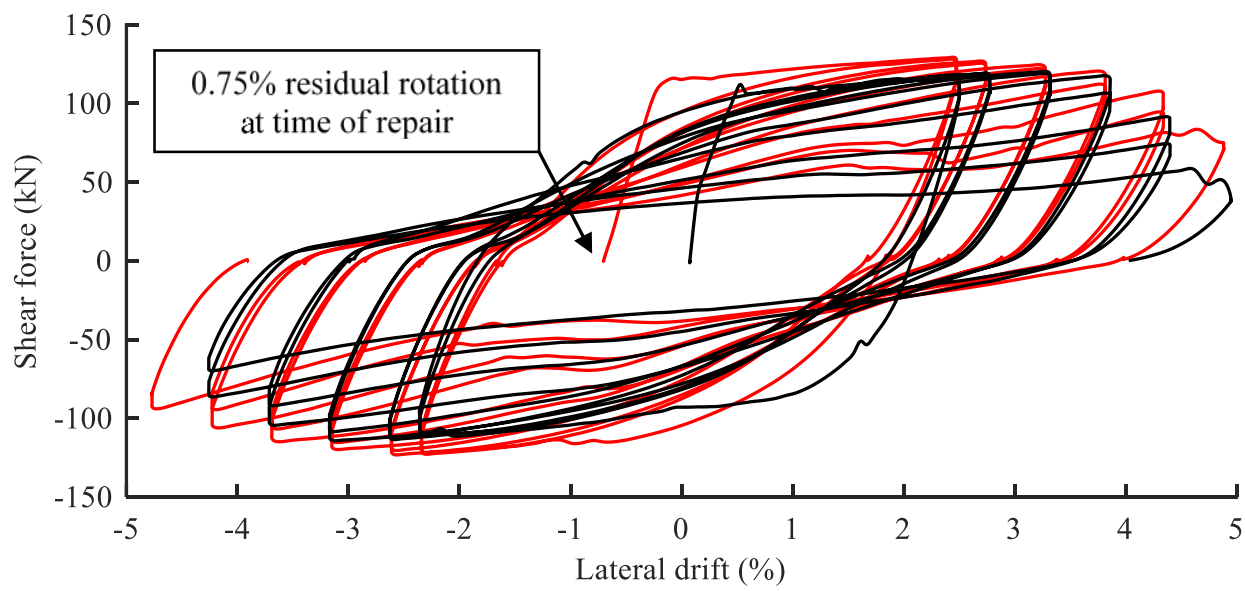

Figure 11: Hysteretic behaviour of repaired (LD-2-R) and undamaged (CYC-NOEQ) nominally identical beam specimens subjected to identical cyclic loading protocols. 
$\checkmark$ Ageing period $<90$ days $\quad$ Ageing period $>90$ days

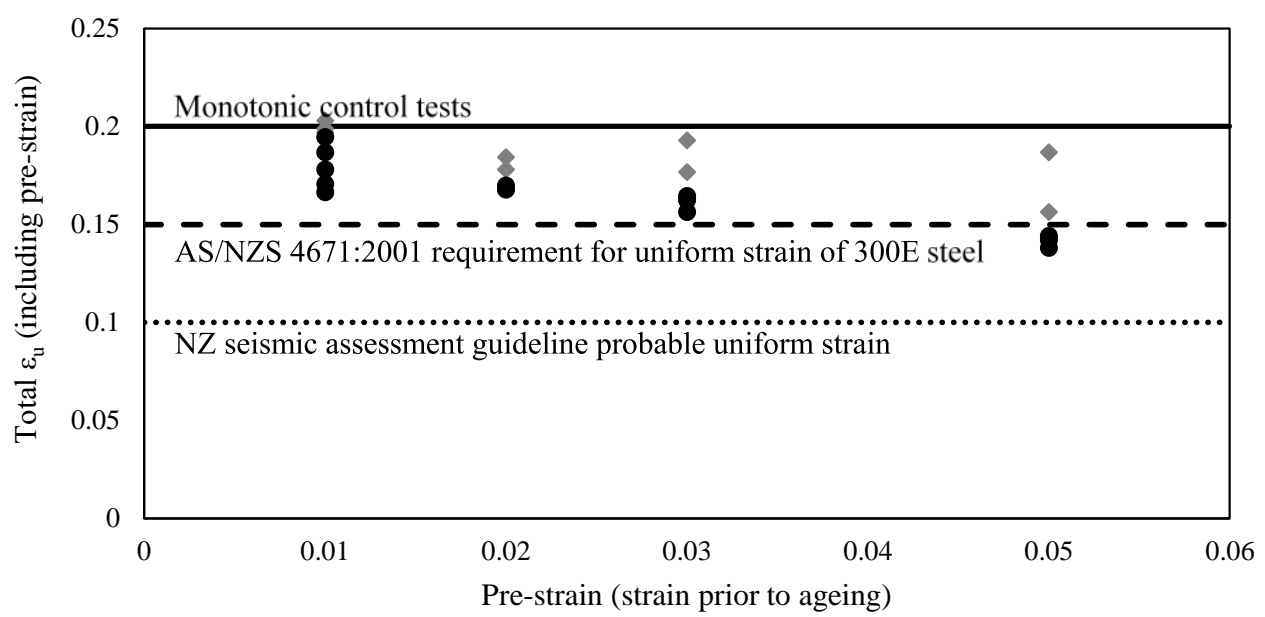

Figure 12: Uniform strain versus pre-strain for strain-aged grade 300E reinforcement. Data taken from Restrepo-Posada et al. [29] and Loporcaro [28].

Previous research has shown that strain ageing can cause reductions in $\varepsilon_{u}$ of up to $30 \%$ [28,29], but the reduction is dependent on the magnitude of strain applied prior to the ageing, as well as the ageing period. Past testing on strain ageing of reinforcement documented in above references has been concentrated on grade $300 \mathrm{E}$ reinforcement used in New Zealand. Fig. 12 shows that the AS/NZS 4671 minimum uniform strain requirement for $300 \mathrm{E}$ reinforcement of 0.15 (90\% probability of exceedance) was still met by all strain-aged tensile coupons except those subjected to a pre-strain of 0.05 and aged for longer than 90 days. The probable uniform strain of 0.1 used in the New Zealand seismic assessment guideline [35] was exceeded in all cases. In practical situations where the pre-strain or reinforcement grade are unknown, in-situ testing is an option to accurately assess the residual strain capacity of strain-aged reinforcement. Alternatively, it may be possible to assume a conservative factor of $0.7 \varepsilon_{u}$ for AS/NZS 4671 steels susceptible to strain ageing. However, an accurate determination of the reduction in strain capacity is unnecessary in many practical instances where reinforcement buckling or concrete crushing is expected to occur prior. Further research is required on steels manufactured to standards other than AS/NZS 4671 [32].

For the purposes of assessing deformation capacity using a moment-curvature and equivalent plastic hinge length approach, the New Zealand seismic assessment guideline [35] stipulates a reinforcement strain limit $\left(\varepsilon_{s . \max }\right)$ of 0.06 . For epoxy-repaired plastic hinges, it would be overly conservative to directly subtract the expected reduction in strain capacity from this limit, as it is not intended to represent the uniform strain of the steel, but rather a practical maximum allowable strain that indirectly accounts for effects such as low-cycle fatigue and axial elongation. Any expected reduction in strain capacity should be subtracted from the lower characteristic value of $\varepsilon_{u}$ for the appropriate steel grade (which can be determined by monotonic testing if the grade is unknown or the value of $\varepsilon u$ is unavailable). The New Zealand seismic assessment guidelines [35] propose the formula $\varepsilon_{s, \max }=0.6 \varepsilon_{u}$ as a method of converting between $\varepsilon_{u}$ and $\varepsilon_{s, \max }$ in cases where $\varepsilon_{u}$ $<0.10$. They also recommend that $\varepsilon_{s, \max }$ be taken as 0.06 for all cases where $\varepsilon_{u}>0.10$.

Residual elongation and strain ageing may have contributed to the early longitudinal fracture that occurred in the Celebi and Penzien [8] specimens, but this cannot be reliably assessed as the relevant data regarding the reinforcement metallurgical characteristics are not available. Further experiments on epoxy repair of elongated plastic hinges with strain-aged reinforcement are required to validate the importance of residual elongation at the time of repair and strain ageing on deformation capacity. Until such data become available, the calculation method described here is considered by the authors to be a rational method of assessing whether there is any increased likelihood of longitudinal reinforcement fracture in epoxy-repaired plastic hinges.

\section{CONCLUSIONS}

Detailed results from three repaired beam tests conducted by the authors were presented. The beams were repaired by epoxy injection and reconstitution of spalled cover concrete following an initial damaging earthquake displacement history loading. As compared with nominally identical (unrepaired) test specimens, the repaired specimens were found to exhibit:

i. increased strengths $(<10 \%$ higher $)$,

ii. reduced secant stiffness to yield values (15 to $25 \%$ lower), iii. increased axial elongation,

iv. longer lengths along which inelastic damage was spread, and

v. comparable energy dissipation and deformation capacities.

Data from these tests, as well as additional relevant data from the literature, were used to derive recommendations on how to quantify the expected response of an epoxy-repaired plastic hinge. The recommendations are based on the limited data available, and are only applicable to ductile frame members exhibiting damage no more severe than flexural cracking (the maximum injected crack width in the beams tested by the authors was $3.5 \mathrm{~mm}$ ), longitudinal reinforcement yielding, and cover concrete delamination (e.g. the damage states shown in Fig. 2). The recommendations are not valid for members exhibiting severe damage characterised by bar buckling or crushing of core concrete. The study yielded the following recommendations:

- Repaired plastic hinges exhibit elongation versus drift responses comparable to what would occur in identical undamaged components and cumulative to any residual elongation at the time of repair. It is recommended that future test programs on epoxy repair report the residual deformations prior to repair in order to further evaluate this conclusion.

- In beams with little or no axial load, a lower-bound postrepair initial secant stiffness to yield can be estimated as $80 \%$ of the stiffness for an identical undamaged component 
(Fig. 6). Repaired columns may exhibit less stiffness restoration; further research is recommended.

- The flexural strength of a repaired ductile plastic hinge is typically at least as high as that of an identical undamaged component

- When considering overstrength of a plastic hinge, it should be recognised that repaired plastic hinges can exhibit flexural strength increases of up to $25 \%$ relative to the strength of identical undamaged components. The authors attribute these strength increases to strain ageing and/or higher levels of strain hardening in the longitudinal reinforcement. The measured residual elongation at the time of repair can be used to calculate an approximate increase in reinforcement strength due to additional strain hardening (Eq. (5)). Further research is required on repaired plastic hinges with strain-aged longitudinal reinforcement; however, a conservative (upper-bound) strain ageing factor of $1.15 f_{y}$ for assessing the overstrength of repaired hinges with reinforcement that is susceptible to strain ageing is recommended until such data are available. These recommended increases in overstrength are in addition to the standard reinforcing steel overstrength factor specified in NZS 3101 [13].

- The post-repair energy dissipation characteristics of repaired plastic hinges are usually comparable to what would occur in identical undamaged components. In cases where severe degradation of the longitudinal reinforcement anchorage occurs, epoxy repair may be unable to fully restore the energy dissipation capacity.

- The post-repair deformation capacity of repaired plastic hinges may or may not be reduced relative to identical undamaged components. If strength increases due to repair force plastic hinge relocation, higher local demands are induced for a given inter-story drift demand. If the plastic hinge does not relocate, reductions in strain capacity of the longitudinal reinforcement, due to strain ageing or residual elongation at the time of repair, has the potential to cause a reduction in deformation capacity in members controlled by tensile fracture of the longitudinal reinforcement. An approach based on moment-curvature analysis and equivalent plastic hinge lengths, e.g. as described in the New Zealand seismic assessment guidelines [35], may be useful for estimating the deformation capacity of repaired plastic hinges in such cases. In the beams tested by the authors, residual elongation at the time of repair was not found to have any effect on deformation capacity. Further research is required.

\section{ACKNOWLEDGMENTS}

The experimental program was funded by the Natural Hazards Research Platform. Atlas Tilt Slab provided assistance with specimen construction, the repair materials were donated by Sika New Zealand, and BBR Contech assisted with execution of the repair. The first author received support from the Commonwealth Scholarship and Fellowship Plan. This project was partially supported by QuakeCoRE, a New Zealand Tertiary Education Commission-funded Centre. This is QuakeCoRE publication number 0323.

\section{REFERENCES}

1 Mahin SA, Bertero VV, Atalay MB and Rea D (1972). "Rate of loading effects on uncracked and repaired reinforced concrete members". Earthquake Engineering Research Center, University of California, Berkeley.

2 Hanson RD (1977). "Repair, Strengthening and Rehabilitation of Buildings: Recommendations for Needed Research". University of Michigan, Department of Civil Engineering, Ann Arbor, MI.
3 Wyllie LA (1978). "Repair of Earthquake Damaged Buildings". IABSE Working Commission Report, ETH Zurich, 30: 65-67.

4 Federal Emergency Management Agency (2012). "Seismic Performance Assessment of Buildings, FEMA P58". Federal Emergency Management Agency, Washington, DC.

5 Applied Technology Council (1998). "Evaluation of Earthquake Damaged Concrete and Masonry Wall Buildings, FEMA 306". Federal Emergency Management Agency, Washington, DC.

6 Henry RS, Dizhur D, Elwood KJ, Hare J and Brunsdon D (2017). "Damage to concrete buildings with precast floors during the 2016 Kaikoura earthquake". Bulletin of the New Zealand Society for Earthquake Engineering, 50(2): 174186.

7 Kam WY, Pampanin S and Elwood K (2011). "Seismic performance of reinforced concrete buildings in the 22 February Christchurch (Lyttleton) earthquake". Bulletin of the New Zealand Society for Earthquake Engineering, 44(4): 239-278.

8 Celebi M and Penzien J (1973). "Hysteretic Behavior of Epoxy-Repaired Reinforced Concrete Beams". Earthquake Engineering Research Center, University of California, Berkeley, USA.

9 Lee DL, Wight JK and Hanson RD (1976). "Original and Repaired Reinforced Concrete Beam-Column SubAssemblages Subjected to Earthquake Type Loading". University of Michigan: Department of Civil Engineering, MI, USA.

10 French CW, Thorp GA and Tsai W-J (1990). "Epoxy repair techniques for moderate earthquake damage". ACI Structural Journal, 87(4): 416-424.

11 Cuevas A and Pampanin S (2017). "Post-Seismic Capacity of Damaged and Repaired Reinforced Concrete Plastic Hinges Extracted from a Real Building". Proceedings of the NZSEE Annual Conference, Wellington, New Zealand.

12 Marder K, Motter C, Elwood K and Clifton GC (2018b). "Testing of seventeen identical ductile reinforced concrete beams with various loading protocols and boundary conditions". Earthquake Spectra, 34(3): 1025-1049. DOI: 10.1193/101717EQS215DP

13 Standards New Zealand (2006). "Concrete Structures Standard, NZS 3101:2006, Incorporating Amendment No. 1,2 , and 3 ". Wellington, NZ.

14 American Concrete Institute (2019). "Building Code Requirements for Structural Concrete, ACI 318-19". American Concrete Institute, Farmington Hills, USA.

15 Marder K, Motter C, Elwood K and Clifton GC (2018a). "Effects of variation in loading protocol on the strength and deformation capacity of ductile reinforced concrete beams". Earthquake Engineering \& Structural Dynamics, 47: 2195-2213. DOI: 10.1002/eqe.3064.

16 Matthews J (2004). "Hollow-core floor slab performance following a severe earthquake". PhD Thesis, University of Canterbury, Christchurch, NZ.

17 Karayannis C, Chalioris C and Sideris K (1998). "Effectiveness of RC beam-column connection repair using epoxy resin injections". Journal of Earthquake Engineering, 2(02): 217-240.

18 Karayannis CG and Sirkelis GM (2008). "Strengthening and rehabilitation of RC beam-column joints using carbonFRP jacketing and epoxy resin injection". Earthquake Engineering and Structural Dynamics, 37(5): 769-790.

19 Marthong C, Dutta A and Deb SK (2013). "Seismic rehabilitation of RC exterior beam-column connections 
using epoxy resin injection". Journal of Earthquake Engineering, 17(3): 378-398.

20 Tsonos AG (2002). "Seismic repair of reinforced concrete beam-column subassemblages of modern structures by epoxy injection technique". Structural Engineering and Mechanics, 14(5), 543-563.

21 Lehman DE, Gookin SE, Nacamuli AM and Moehle JP (2001). "Repair of earthquake-damaged bridge columns". ACI Structural Journal, 98(2): 233-242.

22 Fenwick RC and Megget LM (1993). "Elongation and load deflection characteristics of reinforced concrete members containing plastic hinges". Bulletin of the New Zealand Society for Earthquake Engineering, 26(1): 28-41.

23 Erasmus L and Pussegoda L (1977). "Strain age embrittlement of reinforcing steels". New Zealand Engineering, 32(8): 178-183.

24 Mander J, Panthaki F and Kasalanati A (1994). "Low-cycle fatigue behavior of reinforcing steel". Journal of Materials in Civil Engineering, 6(4): 453-468.

25 Di Ludovico M, Polese M, d'Aragona MG, Prota A and Manfredi G (2013). "A proposal for plastic hinges modification factors for damaged RC columns". Engineering Structures, 51: 99-112.

26 Brooke N and Ingham J (2011). "The Effect of Reinforcement Strength on the Overstrength Factor for Reinforced Concrete Beams". Proceedings of the Ninth Pacific Conference on Earthquake Engineering, Auckland, New Zealand.

27 Davies-Colley S, Kleinjan B, Bull DK and Morris GJ (2015). "Review of Material and Flexural Overstrength Factors for Grade 300E Reinforcing Steel Used in New
Zealand". Proceedings of the NZSEE Annual Conference, Wellington, New Zealand.

28 Loporcaro G (2017). "A least invasive method to estimate the residual strain capacity of steel reinforcement in earthquake-damaged buildings". PhD Thesis, University of Canterbury, Christchurch, NZ.

29 Restrepo-Posada J, Dodd L, Park R and Cooke N (1994). "Variables affecting cyclic behavior of reinforcing steel". Journal of Structural Engineering, 120(11): 3178-3196.

30 Momtahan A, Dhakal R and Rieder A (2009). 'Effects of strain-ageing on New Zealand reinforcing steel bars". Bulletin of the New Zealand Society for Earthquake Engineering, 42(2): 179-186.

31 Erasmus L and Pussegoda L (1980). "The strain aging characteristics of reinforcing steel with a range of vanadium contents". Metallurgical and Materials Transactions A, 11(2): 231-237.

32 Standards Australia (2001). "Steel Reinforcing Materials, AS/NZS 4671:2001". Standards Australia, Sydney, Australia.

33 Standards New Zealand (1982). "Code of Practice for Design of Concrete Structures, NZS 3101:1982". Wellington, New Zealand.

34 Krawinkler H (1992). "Guidelines for Cyclic Seismic Testing of Components of Steel Structures, ATC-24". Applied Technology Council, Redwood City, California.

35 New Zealand Guideline (2017). "The Seismic Assessment of Existing Buildings - Technical Guidelines for Engineering Assessments". Wellington, New Zealand (www.eq-assess.org.nz). 